\title{
Programa de educação em saúde melhora indicadores de autocuidado em diabetes e hipertensão
}

\author{
Health education program improves self-care indicators in diabetes and \\ hypertension
}

\section{El programa de educación en salud mejora los indicadores de autocuidado en diabetes e hipertensión}

\author{
Suelen Magri ${ }^{1, a}$ \\ suelenmagri@gmail.com | https://orcid.org/o000-0002-5043-9113
}

Natalia Weber do Amaral ${ }^{1, b}$

naty bip@hotmail.com | https://orcid.org/oooo-0001-9004-3124

Daniela Novello Martini ${ }^{1, c}$

danielamartini@yahoo.com | https://orcid.org/o0oo-0003-3.524-3654

Luciana Zimmermann Martins dos Santos ${ }^{2, c}$

lucianaz@pmpf.rs.gov.br | https://orcid.org/0000-0002-6992-2148

Luciano de Oliveira Siqueira ${ }^{1, d}$

luciano@upf.br | https://orcid.org/oooo-0002-0415-2226

\footnotetext{
${ }^{1}$ Universidade de Passo Fundo. Passo Fundo, RS, Brasil.

2 Prefeitura Municipal de Passo Fundo. Passo Fundo, RS, Brasil.

a Graduação em Farmácia pela Universidade de Passo Fundo.

b Graduanda em Medicina pela Universidade de Passo Fundo.

c Graduação em Farmácia pela Universidade de Passo Fundo.

d Doutorado em Bioquímica pela Universidade Federal do Rio Grande do Sul.
}

\section{Resumo}

Dentre as doenças crônicas não transmissíveis, a hipertensão arterial sistêmica (HAS) e o diabetes mellitus (DM) são as mais frequentes e constituem um problema de saúde mundial. Diante da necessidade de implantação de programas de autocuidado voltados para essas doenças, o trabalho tem como objetivo validar um programa de autocuidado para pacientes diabéticos e hipertensos. Trata-se de um estudo de coorte prospectivo de uma população de 100 indivíduos que estavam em sala de espera. A coleta dos dados foi realizada por meio de questionários sobre diferentes temas, aplicados nos encontros, para avaliar o conhecimento dos pacientes no pré e no pós-teste. Somente algumas questões específicas de cada tema não apresentaram diferença significativa entre o pré e o pós-teste. No entanto, todos os temas abordados apresentaram incremento de conhecimento no pós-teste. Sendo assim, pode-se validar este programa de autocuidado para pacientes diabéticos e hipertensos, pois promove incremento de conhecimento para os pacientes.

Palavras-chave: Autocuidado; Atenção básica; Doenças crônicas; Educação em saúde; Conhecimento. 


\begin{abstract}
Among chronic non-transmissible diseases, systemic arterial hypertension (SAH) and diabetes mellitus (DM) are the most frequent and constitute a global health problem. In view of the need to implement self-care programs about these diseases, the objective of this study is to validate a self-care program for diabetic and hypertensive patients. It is a prospective cohort study of a population of 100 individuals who were in the waiting room. The data collection was done through questionnaires about different topics, that were applied in the meetings, to evaluate the knowledge of patients in the pretest and posttest. Only a few specific questions of each theme did not present a significant difference between the pretest and posttest. However, all the themes addressed presented posttest knowledge increment. Therefore, this self-care program can be validated for diabetic and hypertensive patients, because it promotes an increase of knowledge for these patients.
\end{abstract}

Keywords: Self-care; Basic attention; Chronic diseases; Health education; Knowledge.

\title{
Resumen
}

Entre las enfermedades crónicas no transmisibles, hipertensión arterial sistémica (HAS) y la diabetes mellitus (DM) son las más frecuentes y constituyen un problema de salud mundial. Ante la necesidad de implantación de programas de autocuidado orientados a estas enfermedades, el trabajo tiene como objetivo validar un programa de autocuidado para pacientes diabéticos e hipertensos. Se trata de un estudio de cohorte prospectiva de una población de 100 individuos que estaban en la sala de espera. La recolección de los datos se realizó a través de cuestionarios sobre diferentes temas, aplicados en los encuentros, para evaluar el conocimiento de los pacientes en el pre-teste y en el post-test. Sólo algunas cuestiones específicas de cada tema no presentaron diferencia significativa entre el pre-test y el post-test. Sin embargo, todos los temas abordados presentaron incremento de conocimiento en el post-test. Por lo tanto, se puede validar este programa de autocuidado para pacientes diabéticos e hipertensos, pues promueve incremento de conocimiento para los pacientes.

Palabras clave: Autocuidado; Atención básica; Enfermedades crónicas; Educación en salud; Conocimiento.

\section{Contribuição dos autores:}

Concepção e desenho do estudo: Luciano de Oliveira Siqueira, Daniela Novello Martini, Luciana Zimmermann Martins dos Santos. Aquisição, análise ou interpretação dos dados: Suelen Magri, Natalia Weber do Amaral, Daniela Novello Martini, Luciana Zimmermann Martins dos Santos.

Redação do manuscrito: Suelen Magri, Natalia Weber do Amaral, Daniela Novello Martini.

Revisão crítica do conteúdo intelectual: Luciano de Oliveira Siqueira.

Declaração de conflito de interesses: não há.

Fontes de financiamento: Programa de Educação pelo Trabalho para a Saúde - PET-SAÚDE/GRADUASUS do Ministério da saúde em conjunto com o Ministério da Educação.

Considerações éticas: baseado em pesquisa registrada e aprovada no Comitê de Ética em Pesquisa, sob o número CAAE: 60764916.8.0000.5342.

Agradecimentos/Contribuições adicionais: ao Ministério da Saúde, Ministério da Educação e Secretaria de saúde do município de Passo Fundo (RS).

Histórico do artigo: submetido: 03 abr. 2019 | aceito: 27 jan. 2020 | publicado: 30 jun. 2020.

Apresentação anterior: não houve.

Licença CC BY-NC atribuição não comercial. Com essa licença é permitido acessar, baixar (download), copiar, imprimir, compartilhar, reutilizar e distribuir os artigos, desde que para uso não comercial e com a citação da fonte, conferindo os devidos créditos de autoria e menção à Reciis. Nesses casos, nenhuma permissão é necessária por parte dos autores ou dos editores. 


\section{Introdução}

As doenças crônicas não transmissíveis (DCNTs) constituem um problema de saúde de escala mundial, representando cerca de $72 \%$ das causas de mortes, cujo aumento da incidência e prevalência está relacionada com a rápida urbanização, transição epidemiológica, transição nutricional, sedentarismo, obesidade, crescimento e envelhecimento da população, e também a maior sobrevida dos indivíduos com DCNTs ${ }^{1-3}$.

Dentre as DCNTs, a hipertensão arterial sistêmica (HAS) e o diabetes mellitus (DM) são as mais frequentes, e seu manejo se dá por mudanças no estilo de vida como atividade física regular, reeducação alimentar e perda de peso. Assim, a orientação adequada sobre o tratamento e o conhecimento das complicações tardias provocadas por essas patologias podem melhorar a qualidade e a expectativa de vida desses pacientes ${ }^{4}$.

Em 2008, cerca de 40\% dos adultos acima de 25 anos foram diagnosticados com hipertensão em todo o mundo, sendo que o número de portadores aumentou de 600 milhões em 1980 para 1 bilhão em 20081. No ano de 2015, a Federação Internacional de Diabetes estimou que 8,8\% da população mundial de 20 a 79 anos de idade (415 milhões de pessoas) vivia com diabetes. Se as tendências atuais persistirem, o número de pessoas com diabetes é projetado para ser superior a 642 milhões em $2040^{3}$.

O diagnóstico de uma doença crônica implica mudanças importantes na vida de seus portadores. A partir dele, os pacientes passam a necessitar de um cuidado integral, envolvendo aspectos biológicos, sociais, econômicos e psicológicos, ocasionando uma percepção de pouco controle acerca da própria vida. Muitas vezes ocorre a rejeição do entendimento por parte dos pacientes sobre as suas doenças, relacionada principalmente com o desconhecimento das complicações tardias, dificultando assim, a adaptação desses indivíduos ao tratamento e à mudança do seu estilo de vida. Portanto, torna-se necessária a realização de técnicas de estímulos e treinamentos para a promoção do autocuidado que promovam mudanças concretas de comportamento dos pacientes, a fim de proporcionar a eles autonomia do cuidado em relação à sua doença ${ }^{3}$.

A falta de escolaridade, somada à linguagem inadequada e às diferentes interações dos profissionais de saúde com os pacientes, relaciona-se diretamente com a baixa adesão das práticas farmacológicas e principalmente das práticas não farmacológicas que são essenciais para o processo de educação em saúde de doenças como DM e HAS, tornando-se assim, uma barreira para o processo do autocuidado. Neste contexto, observa-se a necessidade da execução de ações educativas de fácil condução por meio de profissionais capacitados, que gerem resultados a curto prazo e melhorem a capacidade dos pacientes de autogerenciar suas doenças, adaptando essas práticas aos seus cotidianos, melhorando assim a qualidade de vida desses indivíduos ${ }^{3,4,6,7}$.

Ações realizadas em grupo, como dinâmicas que proporcionam relatos de experiências dos pacientes, permitem um processo integrador entre eles, em que a situação de um paciente ajuda o outro a entender melhor o processo da sua doença e, muitas vezes, auxilia na compreensão das complicações tardias que o DM e HAS causam, alertando os indivíduos para uma maior preocupação com sua saúde³.

Diante da necessidade de implantação de programas de autocuidado com o objetivo de minimizar as complicações tardias, a validação de um programa de autocuidado voltado para as equipes de saúde pode ser útil na medida em que reduz a sobrecarga dos serviços e retrabalho desses profissionais, o que impacta positivamente nos custos em saúde referentes ao tratamento de complicações geradas. Além disso, o presente estudo serve para disseminar conhecimento aos participantes a fim de que estes possam ser multiplicadores para outros indivíduos que sofrem destes mesmos distúrbios. Sendo assim, o estudo tem como objetivo validar um programa voltado ao autocuidado em saúde para pacientes diabéticos e hipertensos. 


\section{Casuística e métodos}

\section{Delineamento do projeto}

Trata-se de um estudo de corte prospectivo de uma população de indivíduos diabéticos e/ou hipertensos, mediante a realização de capacitações para o autocuidado.

\section{Participantes}

Foram selecionados 100 pacientes diabéticos e hipertensos, de ambos os sexos, residentes no bairro Hípica e adjacências na cidade de Passo Fundo-RS, com idades entre 8 e 88 anos (61 anos de mediana), com diferentes níveis de escolaridade e sem distinção de diabéticos do tipo I e II.

\section{Logística}

O projeto foi realizado no período de janeiro a setembro de 2018, em que participantes do estudo foram avaliados no Centro de Atendimento Integrado em Saúde (Cais Hípica - Dr. Erwin Crusius), na cidade de Passo Fundo, RS. Neste CAIS são oferecidos diversos serviços de atendimento à saúde da comunidade, sendo composto por equipes de profissionais da área da saúde englobando enfermagem, técnicos de enfermagem, psicologia, nutrição, fisioterapia, fonoaudiologia, farmácia, odontologia e especialidades médicas, disponibilizando os serviços de acolhimento aberto em saúde mental, serviço de triagem especializado e atendimentos individuais. Também ocorrem atendimentos de acordo com as demandas, atendimento familiar e avaliação dos casos por equipe multiprofissional.

\section{Critérios de inclusão e exclusão}

Foram incluídos neste estudo todos os pacientes com diagnóstico de diabetes e/ou hipertensão, bem como os possíveis cuidadores destes pacientes. Foram excluídos indivíduos não portadores de hipertensão e/ou diabetes, ou que não participaram integralmente da atividade com resultados pré e pós-teste.

\section{Protocolo experimental}

Os pacientes que aguardavam consulta médica na sala de espera da unidade básica foram convidados para participar dos encontros, que ocorriam uma vez por semana. Os pesquisadores elegeram 5 temas principais relevantes para a unidade básica em estudo: fisiopatologia do DM e HAS; cuidados nutricionais para diabéticos e hipertensos; cuidados e adesão farmacológica; saúde bucal e complicações tardias do DM e HAS. Cada tema abordado tinha 4 encontros com participação de aproximadamente 7 pacientes em média, totalizando em torno de 30 pacientes analisados em cada tema. No dia dos encontros, eram realizadas as atividades com os pacientes, divididas em três momentos:

1. Apresentação do tema e aplicação de questionário como um pré-teste para quantificar o conhecimento prévio dos pacientes (duração de \pm 15 minutos). Os questionários aplicados nos encontros estão descritos no quadro 1.

2. Após a aplicação do pré-teste, realizou-se uma roda de conversa envolvendo todos os pacientes, onde foi discutido o tema proposto naquele encontro, com duração entre 30 a 45 minutos. Neste momento, deu-se a possibilidade para os pacientes relatarem suas experiências e esclarecerem suas dúvidas. Como material de apoio, utilizaram-se basicamente recursos humanos e audiovisuais (cartazes, apresentação oral, Datashow) disponíveis na unidade ou cedidos por patrocinadores (folders educativos, por exemplo). 
3. Após o encerramento da discussão do tema, aplicou-se o mesmo questionário com as mesmas perguntas utilizadas anteriormente como um pós-teste para avaliar o conhecimento obtido pelos pacientes durante a atividade (com duração de 15 minutos).

Quadro 1 - Descrição dos questionários aplicados.

\begin{tabular}{|c|c|}
\hline ENCONTROS & QUESTÕES \\
\hline 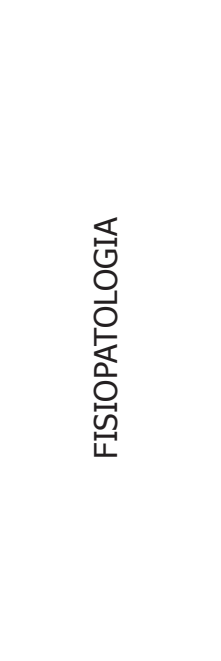 & $\begin{array}{l}\text { 1. Diabetes e pressão alta são doenças para toda a vida? } \\
\text { 2. Quem tem diabetes não controlada e pressão alta pode não ter sintomas? } \\
\text { 3. A diabetes é alta quando for maior que } 140 \mathrm{Omg} / \mathrm{dl} \text { ? E a pressão é alta quando for maior ou } \\
\text { igual a } 14 \text { por } 9 \text { ? } \\
\text { 4. Diabetes e pressão alta podem trazer problemas para o coração, cérebro e rins? } \\
\text { 5. O tratamento para diabetes e pressão alta é para toda vida? } \\
\text { 6. A diabetes e a pressão alta também podem ser tratadas sem remédios? } \\
\text { 7. Exercícios físicos regulares ajudam a controlar a diabetes e a pressão alta? } \\
\text { 8. Para quem é obeso, perder peso ajuda a controlar a diabetes e a pressão alta? } \\
\text { 9. Diminuir o consumo de açúcares e massas ajuda a controlar a diabetes? Diminuir o sal da } \\
\text { comida ajuda a controlar a pressão alta? } \\
\text { 10. Diminuir o nervosismo ajuda a controlar a diabetes e a pressão alta? }\end{array}$ \\
\hline 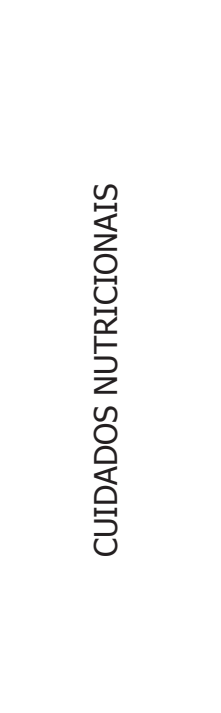 & $\begin{array}{l}\text { 1. Os diabéticos e hipertensos devem ter uma alimentação saudável igual ao que todas as } \\
\text { 2. Éssoas deveriam consumir? } \\
\text { para analisar a quantidade de sódio(sal) e glicose(açúcar)? } \\
\text { 3. O consumo variado de legumes, verduras e frutas ajuda a controlar diabetes e hipertensão? } \\
\text { 4. Pessoas com diabetes não conseguem metabolizar carboidrato (açúcar)? } \\
\text { 5. É importante que as pessoas que têm diabetes e pressão alta façam exercícios físicos de } 30 \\
\text { minutos a } 1 \text { hora por dia na maioria dos dias da semana? } \\
\text { 6. Manter o peso ideal controla o colesterol. Isso é verdade? } \\
\text { 7. O colesterol só faz mal para a saúde? } \\
\text { 8. O colesterol pode causar arteriosclerose (acúmulo de gordura nas artérias)? } \\
\text { 9. Os triglicerídeos são gorduras boas? } \\
\text { 10. Evitar cigarro e bebidas alcoólicas são passos saudáveis? }\end{array}$ \\
\hline
\end{tabular}




\begin{tabular}{|c|c|}
\hline ENCONTROS & QUESTÕES \\
\hline 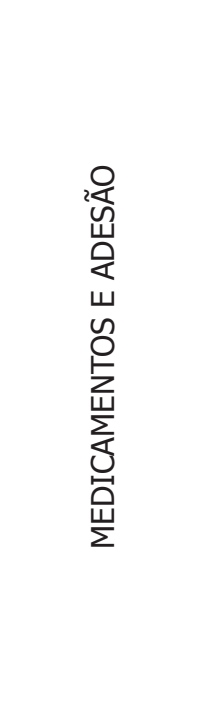 & $\begin{array}{l}\text { 1. O tratamento de diabetes e hipertensão é só pelo uso de medicamentos, sem precisar fazer } \\
\text { 2. Quando o açúcar do sangue estiver baixo, ingerir algo doce melhora? } \\
\text { 3. Medicamento é diferente de remédio? } \\
\text { 4. Para que os medicamentos tenham o efeito desejado, eles devem ser usados de forma } \\
\text { correta e com orientação médica e farmacêutica? } \\
\text { 5. Os medicamentos podem ser partidos ao meio? As cápsulas podem ser abertas? } \\
\text { 6. O medicamento sempre deve ser tomado com água? } \\
\text { 7. Os diabéticos podem tomar qualquer tipo de xarope de farmácia em caso de gripes ou } \\
\text { 8. Todos os medicamentos podem ser tomados a qualquer hora do dia? } \\
\text { 9. Medicamentos que fazem o paciente urinar mais ajudam a diminuir a pressão arterial? } \\
\text { 10. A insulina aumenta o açúcar no sangue? }\end{array}$ \\
\hline 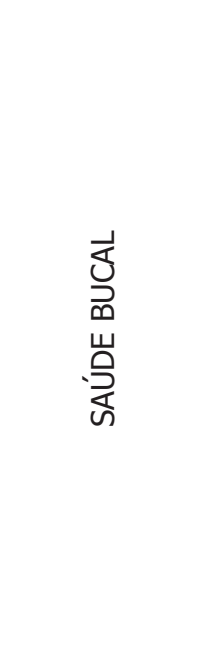 & $\begin{array}{l}\text { 1. Os diabéticos apresentam maior chance de ter infecções de boca? } \\
\text { 2. Pessoas com diabetes podem desenvolver problemas nos dentes e gengivas se o açúcar no } \\
\text { sangue continuar alto? } \\
\text { 3. Gengivas vermelhas, doloridas e com sangramento são os primeiros sinais de gengivite } \\
\text { (inflamação na gengiva)? } \\
\text { 4. A causa principal da gengivite é a má higiene oral? } \\
\text { 5. Enxaguantes bucais e limpeza profissional do dentista podem tratar a gengivite? } \\
\text { 6. Quando o processo se agrava, pode ocorrer perda óssea dos dentes? } \\
\text { 7. A periodontite já é a fase de doença na boca? } \\
\text { 8. É importante que o dentista saiba que seu paciente é hipertenso e/ou diabético? } \\
\text { 9. Diabéticos têm dificuldade de cicatrização e facilidade para hemorragia? } \\
\text { 10. Medicamentos para hipertensão podem causar boca seca? }\end{array}$ \\
\hline 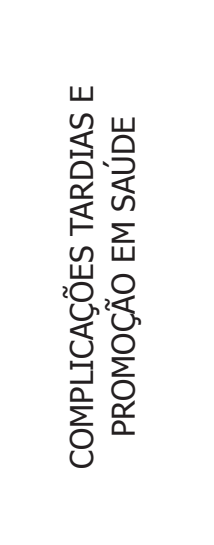 & $\begin{array}{l}\text { 1. Quando a diabetes está alta, o risco de doenças e infecções é maior? } \\
\text { 2. Diabetes e hipertensão podem causar complicações no coração, rins, olhos e pés? } \\
\text { 3. Os rins têm uma função muito importante para a filtração do sangue? } \\
\text { 4. Diabetes alta faz com que os rins filtrem muito sangue, sobrecarregando nossos órgãos? } \\
\text { 5. Pessoas com doença grave nos rins precisam de transplante ou de sessões de hemodiálise? } \\
\text { 6. O dano aos nervos por conta da diabetes pode causar formigamento, dores e pele seca? } \\
\text { 7. Calos não-tratados podem transformar-se em úlceras (feridas abertas)? } \\
\text { 8. O cuidado com os pés do paciente diabético merece atenção? } \\
\text { 9. Glaucoma e catarata podem ser problemas de visão causados pelo diabetes, por exemplo? } \\
\text { 10. Gorduras nas artérias, infarto e derrame podem ser causados pela hipertensão? }\end{array}$ \\
\hline
\end{tabular}

Fonte: Elaborado pelos autores (2018).

\section{Validação dos questionários}

Para validação e elaboração da versão definitiva dos questionários, realizou-se um pré-teste mediante aplicação dos questionários a quinze indivíduos da população do estudo do mesmo modo que seria posteriormente aplicado ao restante do grupo. Ao término da aplicação, os participantes foram questionados 
a respeito de dificuldades na compreensão das perguntas. As dúvidas foram sanadas, e as dificuldades corrigidas na versão definitiva contendo cinco blocos de dez perguntas.

\section{Análise estatística}

Ao término do programa, os questionários foram avaliados de acordo com o conhecimento obtido nos encontros. Os resultados foram reunidos e compilados em uma planilha de trabalho e foram avaliados em medidas de tendência central (média, mediana e moda) e medidas de dispersão (desvio e erro padrão), sendo posteriormente expressos em termos percentuais ou médios. Para determinação de distribuição normal, aplicou-se o teste de Shapiro-Wilk. Por apresentar distribuição paramétrica, aplicou-se o teste t de Student para amostras pareadas. Os resultados foram considerados estatisticamente significativos para $\mathrm{p} \leq \mathrm{0,05}$.

\section{Resultados}

Participaram do estudo 165 pacientes, onde 100 pacientes atenderam os critérios de inclusão e exclusão e foram incluídos no estudo. Destes, 56 eram mulheres, com média de idade de 52,0 $\pm 16,0$ anos e 44 homens, com média de idade de 51,6 $\pm 16,6$ anos. Após a análise dos questionários coletados nos encontros, foi possível identificar categorias de conhecimento do autocuidado dos pacientes: conhecimento da fisiopatologia, nutrição, medicamentos, saúde bucal e complicações tardias.

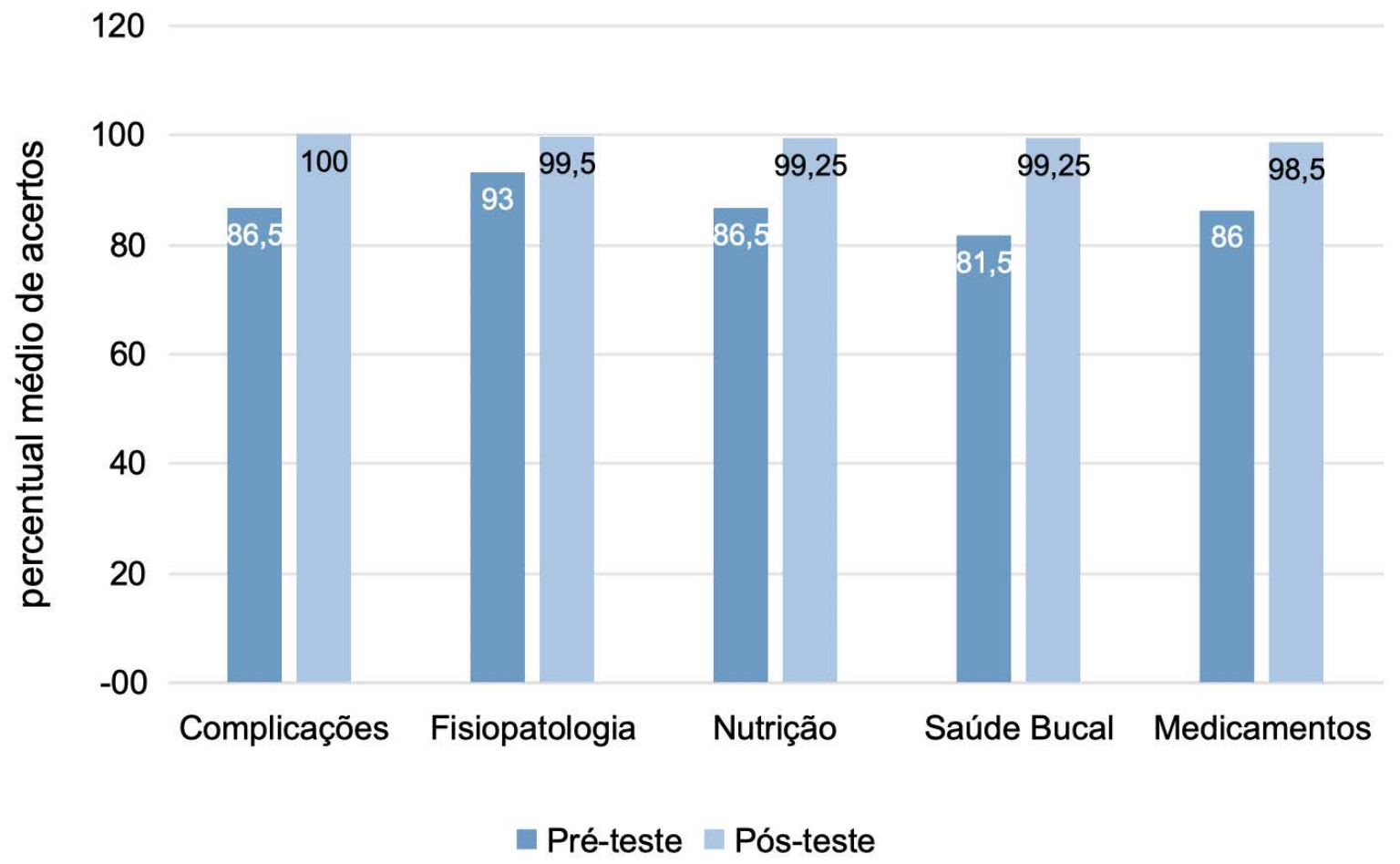

Figura 1 - Percentual médio de acertos das questões do pré-teste e pós-teste em relação a todos os temas abordados nos encontros. Resultados expressos como média \pm desvio padrão

Fonte: Elaborado pelos autores (2018). 
Quadro 2 - Percentual de acerto das questões de 1 a 5 aplicadas em cada tema em relação ao pré-teste e pós-teste. Dados expressos como valor médio

\begin{tabular}{|c|c|c|c|c|c|c|c|c|c|c|c|c|c|c|c|}
\hline & \multicolumn{3}{|c|}{ Questão 1} & \multicolumn{3}{|c|}{ Questão2 } & \multicolumn{3}{|c|}{ Questão 3} & \multicolumn{3}{|c|}{ Questão 4} & \multicolumn{3}{|c|}{ Questão 5} \\
\hline & 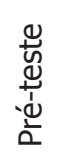 & 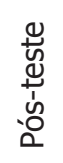 & $\begin{array}{l}0 \\
\frac{0}{0} \\
\frac{0}{0} \\
\frac{0}{3}\end{array}$ & 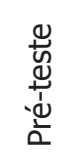 & 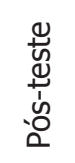 & $\begin{array}{l}0 \\
0 \\
\frac{0}{0} \\
\frac{\overline{0}}{\rho 0}\end{array}$ & 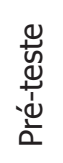 & 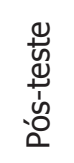 & $\begin{array}{l}0 \\
\frac{0}{0} \\
\frac{\overline{0}}{\pi}\end{array}$ & 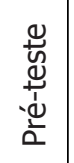 & 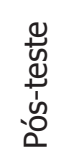 & $\begin{array}{l}0 \\
\frac{0}{0} \\
\frac{\overline{0}}{\pi 0}\end{array}$ & 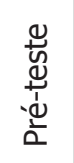 & 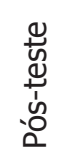 & $\begin{array}{l}\frac{0}{0} \\
\frac{0}{0} \\
\frac{\overline{0}}{\rho}\end{array}$ \\
\hline $\begin{array}{c}\text { Complicações } \\
\text { Tardias }\end{array}$ & 93 & 100 & 0,075 & 95 & 100 & 0,154 & 93 & 100 & 0,075 & 65 & 100 & 0,001 & 88 & 100 & 0,016 \\
\hline Fisiopatologia & 95 & 100 & 0,154 & 85 & 100 & 0,007 & 92 & 100 & 0,075 & 92 & 100 & 0,075 & 97 & 97 & 1,000 \\
\hline Nutrição & 82 & 100 & 0,003 & 100 & 100 & 1,000 & 97 & 100 & 0,324 & 62 & 92 & 0,001 & 100 & 100 & 1,000 \\
\hline Saúde Bucal & 77 & 100 & 0,001 & 75 & 100 & 0,000 & 85 & 100 & 0,007 & 80 & 100 & 0,001 & 80 & 97 & 0,007 \\
\hline Medicamentos & 82 & 100 & 0,003 & 85 & 100 & 0,007 & 72 & 100 & 0,001 & 97 & 100 & 0,324 & 85 & 100 & 0,007 \\
\hline
\end{tabular}

Fonte: Elaborado pelos autores (2018).

Quadro 3 - Percentual de acerto das questões de 6 a 10 aplicadas em cada tema em relação ao pré-teste e pós-teste. Dados expressos como valor médio

\begin{tabular}{|c|c|c|c|c|c|c|c|c|c|c|c|c|c|c|c|}
\hline & \multicolumn{3}{|c|}{ Questão 6} & \multicolumn{3}{|c|}{ Questão 7} & \multicolumn{3}{|c|}{ Questão 8} & \multicolumn{3}{|c|}{ Questão 9} & \multicolumn{3}{|c|}{ Questão 10} \\
\hline & 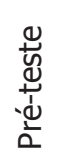 & 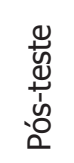 & $\begin{array}{l}0 \\
\frac{0}{0} \\
\frac{\overline{0}}{10} \\
\frac{10}{>}\end{array}$ & 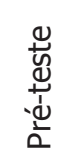 & 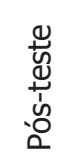 & $\begin{array}{l}0 \\
\frac{0}{0} \\
\frac{\overline{0}}{10} \\
\frac{10}{>}\end{array}$ & 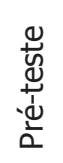 & 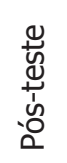 & $\begin{array}{l}0 \\
\frac{0}{0} \\
\frac{\overline{0}}{\pi} \\
\frac{\pi}{>}\end{array}$ & 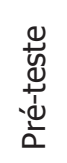 & 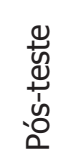 & $\begin{array}{l}0 \\
\frac{0}{0} \\
\frac{0}{0} \\
\frac{0}{5}\end{array}$ & 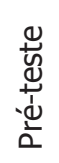 & 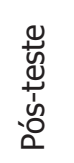 & $\begin{array}{l}0 \\
\frac{0}{0} \\
\frac{0}{0} \\
\frac{0}{3}\end{array}$ \\
\hline $\begin{array}{c}\text { Complicações } \\
\text { Tardias }\end{array}$ & 90 & 100 & 0,036 & 73 & 100 & 0,001 & 85 & 100 & 0,007 & 90 & 100 & 0,036 & 95 & 100 & 0,154 \\
\hline Fisiopatologia & 70 & 97 & 0,001 & 100 & 100 & 1,000 & 100 & 100 & 1,000 & 100 & 100 & 1,000 & 97 & 100 & 0,001 \\
\hline Nutrição & 97 & 100 & 0,324 & 72 & 100 & 0,001 & 95 & 100 & 0,154 & 65 & 100 & 0,001 & 92 & 100 & 0,075 \\
\hline Saúde Bucal & 80 & 100 & 0,001 & 57 & 100 & 0,001 & 97 & 100 & 0,324 & 97 & 100 & 0,324 & 85 & 95 & 0,120 \\
\hline Medicamentos & 95 & 100 & 0,154 & 97 & 100 & 0,324 & 95 & 95 & 1,000 & 82 & 100 & 0,003 & 67 & 90 & 0,003 \\
\hline
\end{tabular}

Fonte: Elaborado pelos autores (2018).

O tema fisiopatologia do DM e HAS mostra que a média de acerto global passou de $93 \%$ para $100 \%$, apresentando um aumento estatisticamente significante $(\mathrm{p}<0,05)$. Na análise individual das questões, somente as questões 1 e 5 não mostraram diferença estatística significante. As questões 7, 8 e 9 apresentaram média de $100 \%$ no pré e pós-teste, indicando que o conhecimento prévio sobre estas questões está claro e satisfatório para a população analisada, mas, ao mesmo tempo, houve um ganho de aprendizado em 70\% das questões aplicadas.

Em relação ao tema cuidados nutricionais a análise das médias das notas mostra um incremento estatisticamente significante de $87 \%$ para 99\%. A análise individual das questões mostra que as perguntas 2, 3, 5, 6, 8 e 10 não mostraram diferença significante. A questão 4 (Pessoas com diabetes não conseguem metabolizar carboidratos (açúcares)?) e 9 (Os triglicerídeos são gorduras boas?) mostram um percentual de acerto menor que $70 \%$ no pré-teste, o que indica baixo conhecimento sobre o assunto das questões específicas. 
No pós-teste, porém, os percentuais de acerto foram de 92\% e 100\% (respectivamente), demonstrando que o conhecimento que era baixo passou a ser praticamente pleno para os pacientes analisados. As outras questões não apresentaram diferença significativa, sendo que as perguntas 2 e 5 apresentaram média de $100 \%$ tanto no pré quanto no pós-teste, demonstrando que esses pacientes apresentam conhecimento prévio satisfatório sobre essas questões. O resultado mostra que o grau de incremento de conhecimento esteve presente em $40 \%$ das questões, corroborando o fato de que o conhecimento prévio dos pacientes sobre cuidados nutricionais era relativamente satisfatório.

A análise dos resultados de conhecimento de cuidados sobre saúde bucal apresentou uma elevação estatisticamente significante da nota média, tendo um aumento no percentual de acerto de $82 \%$ para $99 \%$. A análise individual das questões mostra que as questões 8, 9 e 10 não mostraram diferença significante, indicando um incremento de aprendizado em torno de 70\% das questões aplicadas.

A análise estatística dos resultados relacionados a complicações tardias mostra um incremento estatisticamente significante de $87 \%$ para $100 \%$ de acerto. A análise individual das questões relativas às complicações mostra que as perguntas 4, 5, 6 e 10 apresentaram um incremento estatisticamente significante, sendo que as questões 7,8 e 9 não mostraram diferença estatística, pois o conhecimento prévio das questões abordadas foi de $100 \%$ tanto no pré-teste quanto no pós-teste.

A análise dos resultados sobre o uso de medicamentos mostra um aprimoramento estatisticamente significante do conhecimento de $86 \%$ para $99 \%$. Ao analisar individualmente as questões relativas a medicação, verifica-se que todas as perguntas mostraram um incremento estatisticamente significante, exceto as de número 4, 6, 7 e 8, indicando uma aquisição de aprendizado em torno de 60\% das questões aplicadas.

De acordo com a análise do percentual médio de acertos do pré-teste em relação ao pós-teste, todos os temas apresentaram diferença significativa, indicando que o programa de autocuidado melhorou o conhecimento dos pacientes em relação aos seus conhecimentos prévios sobre todos os temas.

A figura 2 representa a validade do protocolo aplicado, avaliando o número de questões que apresentaram incremento de conhecimento para os pacientes, ou seja, o número de questões que aumentaram a nota no pós-teste. No tema sobre complicações tardias, $100 \%$ das questões que foram aplicadas nos encontros mostraram aumento da nota no pós-teste, no entanto, a análise estatística demostra que a diferença foi significante em $60 \%$ das questões aplicadas. Em relação ao tema fisiopatologia, 60\% (6 das 10 questões) tiveram um aumento da nota, com 40\% das questões apresentando diferença estatística significativa.

$\mathrm{Na}$ análise dos resultados do tema sobre nutrição, 90\% das questões aplicadas aos participantes do programa apresentaram um incremento de conhecimento, sendo que 40\% das questões apresentaram um incremento de conhecimento estatisticamente significativo. Com relação ao tema saúde bucal e medicamentos, ambos obtiveram um percentual de incremento de conhecimento de $100 \%$, no entanto, no tema saúde bucal, a análise estatística demonstra que a diferença foi significante em $70 \%$ das questões, e no tema sobre medicamentos, $60 \%$ das questões apresentaram diferença estatística significativa. 
$120 \%$

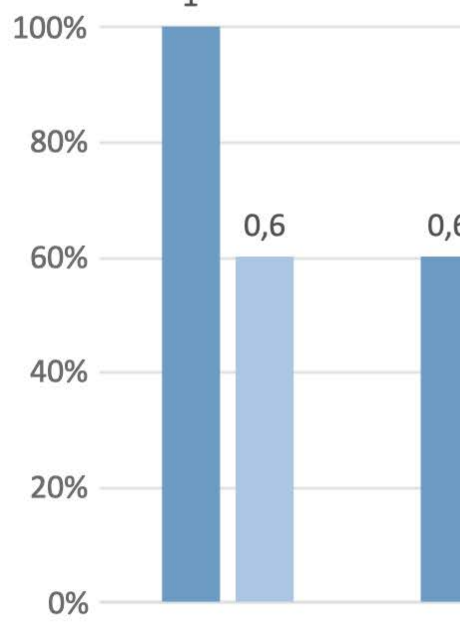

1

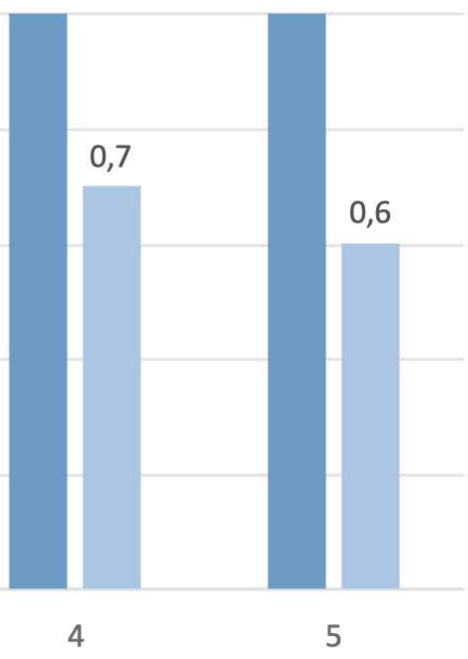

Série1 Série2

Figura 2 - Percentual do número de questões com incremento de conhecimento e percentual do número de questões com diferença significativa no incremento de conhecimento Fonte: Elaborado pelos autores (2018).

A figura 3 traz o percentual de aumento de conhecimento dos diferentes temas discutidos nos encontros. Os resultados mostram que o conhecimento prévio sobre fisiopatologia é o mais adequado, pois o percentual de incremento foi relativamente baixo (8\%), o que mostra que o protocolo aplicado pouco acrescentou para o conhecimento, ou que o conhecimento da população já era satisfatório em relação ao tema, antes mesmo da aplicação do protocolo.

Por outro lado, o menor conhecimento avaliado da população foi em saúde bucal, pois o protocolo contribuiu com um incremento de $21 \%$ no conhecimento prévio dos pacientes a respeito deste tema.

Com relação aos temas complicações tardias e medicamentos, verificou-se em ambos um percentual médio de $15 \%$ de incremento de conhecimento após a aplicação do programa. E no tema sobre nutrição o percentual de incremento de conhecimento foi de $14 \%$, demonstrando que a aplicação dos protocolos resultou em aumento do conhecimento para essa população analisada, porém já existia um conhecimento prévio mínimo desses pacientes. 


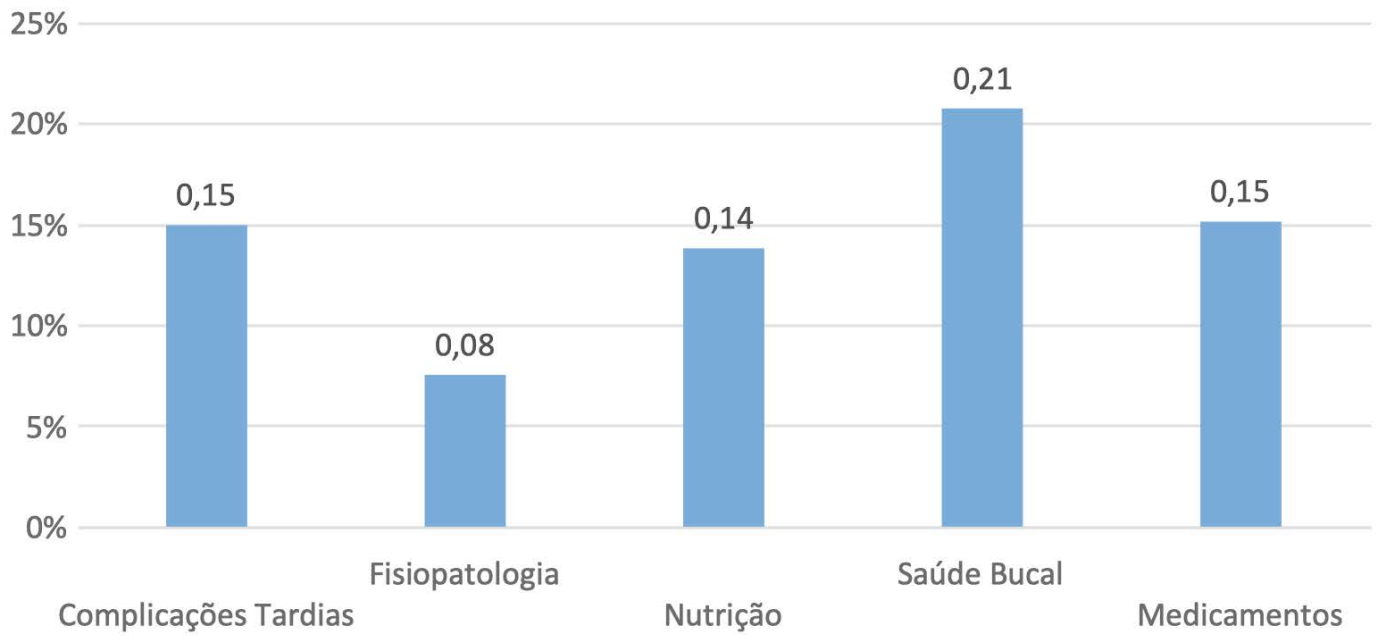

$\%$ médio de incremento de conhecimento

Figura 3 - Percentual médio de incremento de conhecimento das questões sobre os temas Fonte: Elaborado pelos autores (2018).

\section{Discussão}

Os dados do presente estudo apresentam melhorias no autoconhecimento sobre as doenças dos pacientes que participaram da pesquisa, mesmo que, no início do programa muitos pacientes já apresentassem conhecimento satisfatório no pré-teste, como é o caso do tema fisiopatologia do diabetes e hipertensão, no qual houve um incremento de conhecimento de apenas $8 \%$. Fato que difere de um estudo semelhante realizado em uma unidade básica de saúde em uma cidade do interior paulista, São Paulo, Brasil, em que o nível de autoconhecimento dos pacientes foi considerado insatisfatório no pré-teste para a maioria dos indivíduos, o que estava diretamente relacionado à baixa escolaridade dos pacientes participantes daquela pesquisa $^{8}$.

Observa-se no presente estudo que a maioria dos pacientes tem conhecimento sobre o tema medicamentos, sendo que o estudo apresentou um incremento de $15 \%$ no conhecimento desses pacientes, devido, principalmente, ao desconhecimento de questões específicas sobre o mecanismo dos medicamentos, principalmente da insulina que utilizam, seus efeitos colaterais e a diferença entre um medicamento de um remédio. A percepção da maioria dos indivíduos é que os medicamentos são os principais protagonistas no processo de tratamento de sua doença, o que corrobora com o estudo transversal desenvolvido no Ambulatório de Diabetes de uma unidade de atenção terciária, no qual observou-se que independentemente dos medicamentos prescritos, os pacientes com DM e HAS desempenham, com maior frequência, a atividade do autocuidado em relação aos medicamentos que eles utilizam, do que relacionadas às mudanças em seu estilo de vida ${ }^{5}$.

Outros estudos também observaram que, para os portadores de DM e HAS, o autocuidado está relacionado com o fato de fazer o uso correto dos medicamentos, pois geralmente esses pacientes apresentam dificuldades em controlar sua doença com medidas não medicamentosas9-10. Com a percepção de que a prática medicamentosa é fundamental para esses pacientes, pressupõe-se que a orientação dos profissionais da saúde em relação aos medicamentos que são prescritos otimizem o tratamento e melhorem os níveis de glicemia e pressão arterial desses pacientes ${ }^{11}$.

De acordo com a questão relacionada ao exercício físico, $100 \%$ dos pacientes entrevistados reconhecem a importância de que diabéticos e hipertensos pratiquem exercícios físicos de 30 minutos a 1 hora por 
dia, preferencialmente todos os dias da semana, porém a maioria dos pacientes relatam que não praticam exercícios físicos. Resultados semelhantes foram encontrados em alguns estudos com indivíduos portadores de DM atendidos em uma Unidade Básica de Saúde de Várzea Grande - Mato Grosso e pacientes com DM atendidos na Unidade Básica de Saúde de Porteirinha - Minas Gerais, sendo que em ambos os estudos, os pacientes reconhecem a importância de realizar atividades físicas, no entanto, 84,6\% e 79,0\% deles (respectivamente) não praticam ${ }^{7,12}$.

Em um estudo desenvolvido no Ambulatório de Diabetes, os pacientes apresentavam bom conhecimento sobre a restrição dos açúcares e gorduras ainda no pré-teste, pois este item mostrou conhecimento satisfatório5. Na amostra analisada no presente estudo, os pacientes desmontaram um conhecimento satisfatório na maioria das questões sobre nutrição, porém não souberam identificar quais são as gorduras boas e ruins que se deve consumir. Em relação aos triglicerídeos e a importância do colesterol 'bom', apenas entendem que essa gordura é um ponto negativo da alimentação. O percentual de acerto dessas questões no pré-teste foi abaixo de $70 \%$, porém houve um incremento de conhecimento significativo quando foram analisadas no pós-teste, obtendo-se uma melhora no conhecimento dos pacientes sobre essas questões específicas. Em relação ao percentual médio de todas as questões, houve um incremento de conhecimento de 14\%, sendo assim, observa-se que a educação alimentar é um ponto essencial para o controle metabólico para os pacientes com DM e HAS. Entretanto, modificar os hábitos alimentares representa um ponto desafiador no processo do autocuidado ${ }^{13}$.

Analisando o tema saúde bucal, constatou-se que os pacientes possuíam um conhecimento limitado no pré-teste quanto a algumas questões, sendo que o percentual médio de acertos passou de $82 \%$ no pré-teste para 99\% no pós-teste, tratando-se do tema que apresentou o maior percentual médio de incremento de conhecimento (21\%). Desse modo, observa-se que os pacientes com DM e HAS avaliados não possuíam conhecimento necessário em relação à saúde bucal, sendo que essa condição é fundamental no processo de autocuidado, já que os problemas de saúde bucal estão relacionados também aos problemas de doenças cardiovasculares, nutricionais e psicossociais14. A saúde bucal faz parte da saúde em geral e é um fator relevante principalmente para um envelhecimento saudável dos pacientes com DM e HAS, no entanto, a importância deste tema ainda não é devidamente reconhecida ${ }^{15}$.

Em relação ao tema complicações tardias, o incremento de conhecimento foi de $15 \%$, sendo que os pacientes apresentavam conhecimento satisfatório sobre as complicações que o DM e HAS podem causar. Porém, os pacientes analisados apresentaram pouco conhecimento em algumas questões específicas relacionadas à função dos rins e ao cuidado com os calos que podem se transformar em úlceras. Esses dois temas podem prevenir problemas nos rins ou futuras amputações desses pacientes, pois as complicações decorrentes do DM e HAS estão diretamente relacionadas ao conhecimento sobre a doença16. O controle inadequado das doenças crônicas analisadas, como DM e HAS, por um longo período de tempo, é associado a complicações como retinopatia, nefropatia, doenças cardiovasculares e lesões nos pés13. Em um estudo descritivo realizado na Unidade Básica de Saúde da Família no município de Várzea Grande - Mato Grosso, verificou-se que a maioria dos indivíduos examina seus pés diariamente, mas não sabem a importância de tal prática ${ }^{12}$.

Dentro das necessidades de realização de programas educativos voltados ao cuidado com pacientes diabéticos, dois trabalhos foram desenvolvidos no ano de 2009 em Minas Gerais. Ambos os estudos realocaram pacientes para acompanhamento individual e para educação em grupo, sendo que, na forma individual, a avaliação de conhecimento obtido se deu por meio de questionário específico sobre o tema seguido por orientações quanto à qualidade de vida, e nos grupos operativos, por meio de atividades lúdicas17-18. Ambas as estratégias do programa educativo demonstraram perfis semelhantes. Entretanto, a educação em saúde realizada em grupo mostrou resultados mais efetivos do controle glicêmico do que a estratégia individual. Em outro estudo realizado em uma Estratégia Saúde da Família (ESF) em Montes 
Claros, Minas Gerais, na percepção do paciente portador de DM e HAS, a troca de experiências entre si já promove uma reflexão sobre o estilo de vida, o que auxilia na busca de sua própria autonomia ${ }^{19}$.

Em uma revisão sistemática realizada no ano de 2013, com o objetivo de analisar as formas de intervenção voltadas para a educação em saúde de pacientes diabéticos, discute-se os modelos de tratamento existentes e se relata que, para a eficácia dele, o paciente precisa assumir mudanças no estilo de vida, capacidade de identificar, resolver e procurar auxílio de acordo com as necessidades de sua doença. Isso também é reforçado pela American Diabetes Association (ADA), em que "todos os pacientes com DM deveriam receber educação para o autocuidado”, uma vez que com o conhecimento sobre sua doença, os resultados clínicos são melhores20 (p.401).

Em alguns estudos, há uma discussão acerca da baixa adesão aos processos de autocuidado, problematizando com a ideia de que os fatores pessoais, socioeconômicos, culturais e fisiopatológicos da doença e aspectos do sistema de saúde podem influenciar no autogerenciamento do processo21. Em contrapartida a este fato, outro estudo demonstrou que as orientações dadas ao paciente, o incentivo ao estilo de vida saudável e acompanhamento dos portadores com vista ao desenvolvimento de atividades educativas podem sensibilizar os pacientes para o cuidado em saúde ${ }^{22}$.

O presente estudo se limita pelo fato de que a investigação sobre a relação entre as atividades de autocuidado pode ter sofrido interferências com relação à idade dos pacientes, a capacidade de interpretarem as perguntas e o desinteresse em participar dos encontros, o que pode ter facilitado ou dificultado a mudança nas atitudes do autocuidado.

Apesar de haver outros estudos semelhantes, este estudo tem caráter inovador devido à abordagem de educação do autocuidado para pacientes diabéticos e hipertensos. Comprova-se um aprimoramento do autoconhecimento de fatores não modificáveis, mas principalmente dos modificáveis, que podem proporcionar hábitos de vida mais saudáveis para esses pacientes, principalmente devido à maioria dos pacientes participantes serem idosos. A população idosa necessita de uma atenção especial, pois além de apresentarem uma saúde mais frágil, o acometimento por esta condição prejudica a autonomia, funcionalidade e o ritmo de vida destes pacientes. Nesta perspectiva, o paciente idoso portador de diabetes mellitus deve ser informado sobre a importância do autocuidado para um tratamento efetivo ${ }^{7}$, prevenindo complicações mais graves, que por consequência saturam os serviços de atendimento, além de gerar relevante impacto econômico e social.

\section{Conclusão}

A análise dos resultados permite concluir um evidente incremento no conhecimento dos pacientes no pós-teste, sugerindo que há relação entre a educação do autocuidado e o incremento de conhecimento para a realização de mudanças no estilo de vida. Sendo assim, pode-se validar este programa voltado ao autocuidado em saúde para pacientes portadores de DM e HAS, bem como multiplicá-lo e aprimorá-lo em outras unidades de saúde.

A proposta permitiu conscientizar e qualificar o autocuidado de portadores de DM e HAS sobre a importância dos temas abordados nos encontros, auxiliando na prevenção das complicações decorrentes das doenças e melhora na qualidade de vida desses pacientes. Como resultado, espera-se minimizar as complicações tardias e desafogar o sistema de saúde mediante resolução de intercorrências mais simples sem a necessidade de busca no serviço especializado.

Desse modo, propõe-se dar continuidade aos estudos, reavaliando estes mesmos pacientes após um ano com vistas a avaliar a consolidação do conhecimento gerado a longo prazo. 


\section{Referências}

1. World Health Organization. A global brief on hypertension [Internet]. Geneva; 2013 [cited 2018 maio 15]. Available from: http://apps.who.int/iris/bitstream/handle/10665/79059/WHO DCO WHD 2013.2 eng.pdf;jsessionid=FFF18091A211E449149FA1B249A13CA4? sequence $=1$.

2. Grechi EFM, Pascon DM. Conhecimento dos pacientes portadores de diabetes mellitus tipo 2 inseridos no Programa de Saúde ao Adulto. Rev. Fac. Ciênc. Méd. Sorocaba [Internet]. 2016 dez [citado 2018 nov. 15];18(4):204-209. Disponível em: http://revistas.pucsp.br/index.php/RFCMS/article/view/23746/pdf.

3. Oliveira JEP, Montenegro Junior RM, Vencio S, organizadores. Diretrizes da Sociedade Brasileira de Diabetes 2017-2018 [Internet]. São Paulo:Editora Clannad; 2017 [citado 2018 nov. 15]. Disponível em: https://www.diabetes.org.br/ profissionais/images/2017/ diretrizes/diretrizes-sbd-2017-2018.pdf.

4. Miranzi SSC, Ferreira FS, Iwamoto HH, Pereira GA, Miranzi MAS. Qualidade de vida de indivíduos com diabetes mellitus e hipertensão acompanhados por uma equipe de saúde da família. Rev Texto Contexto Enferm [Internet], 2008 dez [citado 2018 jun. 09];17(4):672-679. doi: http://dx.doi.org/10.1590/s0104$\underline{07072008000400007 .}$.

5. Coelho ACM, Boas LCGV, Gomides DS, Freitas MCF, Pace AE. Self-care activities and their relationship to metabolic and clinical control of people with diabetes Mellitus. Rev Texto Contexto Enferm [Internet], 2015 ago [cited 2018 out. 14];24(3):697-705. Disponível em: http://www.scielo.br/pdf/tce/v24n3/ pt 0104-0707-tce-2015000660014.pdf.

6. Imazu MFM, Faria BN, Arruda GO, Sales CA, Marcon SS. Effectiveness of individual and group interventions for people with type 2 diabetes. Rev. Latino-Am. Enfermagem [Internet], 2015 abr [cited 2018 nov. 18];23(2):200-207. Disponível em: http://www.scielo.br/pdf/rlae/v23n2/pt 0104-1169rlae-23-02-00200.pdf.

7. Dias EG, Pardim ACS, Antunes LP, Silva IO, Alves JCS, Jorge SA. Desafios da prática do autocuidado do idoso portador de diabetes mellitus tipo 2. Rev Sustinere [Internet]. 2017 jul [citado 2018 out. 14];5(1):40-50. Disponível em: https://www.e-publicacoes.uerj.br/index.php/sustinere/article/ view/26483/21150.

8. Rodrigues FFL, Santos MA dos, Teixeira CRS, Gonela JT, Zanetti ML. Relação entre conhecimento, atitude, escolaridade e tempo de doença em indivíduos com diabetes mellitus. Acta Paul Enferm. [Internet], 2012 out [citado 2018 jun. 09];25(2):284-90. Disponível em: http://www.scielo.br/pdf/ape/ v25n2/a20v25n2.pdf.

9. Farias HTG. Desafios para atenção em saúde: adesão ao tratamento e controle metabólico em pessoas com diabetes melitus tipo 2, no município de Passos - MG [tese]. Riberão Preto: Universidade de São Paulo; 2011 [citado 2018 nov. 15]. Disponível em: http://www.teses.usp.br/teses/disponiveis/22/22132/ tde08082011\%20155145/publico/HELOISATURCATTOGIMENESFARIA.pdf.

10. Oliveira MSN, Almeida GBS, Chagas DNPC, Salazar PR, Ferreira LV. Autocuidado de idosos diagnosticados com hipertensão arterial e/ou diabetes mellitus. Rev. Enferm UFSM. [Internet], 2017 out [citado 2018 out. 14];7(3)490-500. Disponível em: https://periodicos.ufsm.br/reufsm/article/view/26344/pdf.

11. Visentin A, Mantovani MF, Caveião C, Hey AP, Scheneider EP, Paulinho V. Autocuidado de usuários com diabetes tipo 1 em uma unidade básica de saúde. Rev Enferm UFPE on line [Internet]. 2016 mar [citado 2018 nov. 18];10(3):991-998. Disponível em: http://www.revista.ufpe.br/revistaenfermagem/index.php/ revista/index.

12. Pereira JG, Cardoso MI, de Moraes MAM. Práticas de autocuidado desenvolvidas por diabéticos tipo 2 em uma unidade básica de saúde da família. Rev. G\&S [Internet]. 2017 jul [citado 2018 abr. 08];2(1):11623. Disponível em: https://periodicos.unb.br/index.php/rgs/article/view/30.

13. Sociedade Brasileira de Diabetes (BR). Diretrizes da Sociedade Brasileira de Diabetes. São Paulo: AC Farmacêutica. 2018.

14. Bez AS. Resiliência e sua relação com mudança na autopercepção de saúde bucal em idosos independentes do sul do Brasil [dissertação]. Porto Alegre: Universidade Federal do Rio Grande do Sul; 2013. [citado 2018 out. 14]. Disponível em: http://bit.ly/2gCimVI.

15. Simões ACA, Oliveira RS, Carvalho DM. O envelhecimento no contexto da odontologia. Revista Triângulo [Internet]. 2009 [citado 2018 abr. 09];2(1):43-52. Disponível em: http://bit.ly/2fIOTuz. 
16. Dizaji MB, Taghdisi MH, Solhi M, Hoseini SM, Shafieyan Z, Qorbani M. Effects of educational intervention based on PRECEDE model on self-care behaviors and control in patients with type 2 diabetes in 2012. J Diabetes Metabol Disorders [Internet]. 2014 [cited 2018 out. 14];13:72. Disponível em: https://www. ncbi.nlm.nih.gov/p mc/articles/PMC4114427/pdf/2251-6581-13-72.pdf.

17. Torres HC, Franco LJ, Stradioto MA, Hortale VA, Schall VT. Avaliação estratégica de educação em grupo e individual no programa educativo em diabetes. Rev Saúde Pública [Internet]. 2009 [citado 2018 jun. 06];43(2):291-298. Disponível em: http://www.scielo.br/pdf/rsp/v43n2/05.pdf.

18. Pereira FRL, Torres HC, Cândido NA, Alexandre LR. Promovendo o autocuidado em diabetes na educação individual e em grupo. Cienc Cuid Saúde [Internet]. 2009 [citado 2018 jun. 10];8(4):594-599. Disponível em: http://ojs.uem.br/ojs/index.php/CiencCuidSaude/article/viewFile/9686/5391.

19. Almeida ER, Moutinho CB, Leite MTS. A prática da educação em saúde na percepção dos usuários hipertensos e diabéticos. Saúde em Debate [Internet]. 2014 [citado 2018 nov. 18];38(101):328-336. Disponível em: http://www.scielo.br/pdf/sdeb/v38n101/0103-1104-sdeb-38-101-0328.pdf.

20. Grillo MFF, Neumann CR, Scain SF, Rozeno RF, Gross JL, Leitão CB. Efeito de diferentes modalidades de educação para o autocuidado a pacientes com diabetes. Rev Assoc Med Bras. [Internet] 2013 [citado 2018 jun. 09];59(4):400-405. Disponível em: http://www.scielo.br/pdf/ramb/v59n4/v59n4a21.pdf.

21. Neta DSR, Silva ARV, Silva GRF. Adesão das pessoas com diabetes mellitus ao autocuidado com os pés. Rev Bras Enferm [Internet]. 2015 fev [citado 2018 jun. 10];68(1)111-116. Disponível em: http://www. scielo.br/pdf/reben/v68n1/0034-7167-reben-68-01-0111.pdf.

22. Morais GFC, Soares MJGO, Costa MMLC, Santos IBC. O diabético diante do tratamento, fatores de risco e complicações crônicas. Rev Enferm. UER] [Internet] 2009 [citado 2018 jun. 10];17(2):240-245. Disponível em: http://www.facenf.uerj.br/v17n2/v17n2a18.pdf. 